Publ. Mat. 47 (2003), 3-29

\title{
THE FOURIER TRANSFORM IN WEIGHTED LORENTZ SPACES
}

\author{
Gord SinNAMON
}

\begin{abstract}
Necessary conditions and sufficient conditions on weights $u$ and $w$ are given for the Fourier transform $\mathcal{F}$ to be bounded as a map between the Lorentz spaces $\Gamma_{q}(w)$ and $\Lambda_{p}(u)$. This may be viewed as a weighted extension of a result of Jodeit and Torchinsky on operators of type $(1, \infty)$ and $(2,2)$. In the case $0<p \leq 2=q$, the necessary and sufficient conditions are equivalent and give a simple weight condition which is equivalent to $\mathcal{F}: \Gamma_{2}(w) \rightarrow \Lambda_{p}(u)$ and also to $\mathcal{F}: \Gamma_{2}(w) \rightarrow \Gamma_{p}(u)$.
\end{abstract}

\section{Introduction}

The Fourier transform on $\mathbf{R}^{n}$ is bounded as a map from $L^{1}$ to $L^{\infty}$ and also as a map from $L^{2}$ to $L^{2}$. We say it is of type $(1, \infty)$ and $(2,2)$. In [8], Jodeit and Torchinsky showed that a map $T$ is of type $(1, \infty)$ and $(2,2)$ if and only if there is a constant $D$ such that

$$
\int_{0}^{z}(T f)^{*}(x)^{2} d x \leq D \int_{0}^{z}\left(\int_{0}^{1 / t} f^{*}\right)^{2} d t, \quad f \in L^{1} \cap L^{2}, z>0 .
$$

Here $f^{*}$ denotes the non-increasing rearrangement of $f$. We give weighted extensions of this result and interpret them as boundedness properties of the operator $T$ between weighted Lorentz spaces. If $p \in(0, \infty)$ and $v$ is a non-negative weight we define the Lorentz space $\Lambda_{p}(v)$ to be the collection of functions $f: \mathbf{R}^{n} \rightarrow \mathbf{R}$ for which

$$
\|f\|_{\Lambda_{p}(v)}=\left(\int_{0}^{\infty}\left(f^{*}\right)^{p} v\right)^{1 / p}
$$

2000 Mathematics Subject Classification. Primary: 42B35; Secondary: 46E30.

Key words. Fourier transform, weights, Lorentz space.

Support from the Natural Sciences and Engineering Research Council of Canada is gratefully acknowledged. 
is finite. Define $f^{* *}$ by $f^{* *}(t)=\frac{1}{t} \int_{0}^{t} f^{*}$ and let $\Gamma_{p}(v)$ be the collection of those $f$ for which

$$
\|f\|_{\Gamma_{p}(v)}=\left(\int_{0}^{\infty}\left(f^{* *}\right)^{p} v\right)^{1 / p}
$$

is finite.

Our object is to investigate inequalities of the form

$$
\begin{aligned}
& \left(\int_{0}^{\infty}(T f)^{*}(x)^{q} u(x) d x\right)^{1 / q} \\
& \quad \leq C\left(\int_{0}^{\infty}\left(\int_{0}^{1 / t} f^{*}\right)^{p} v(t) d t\right)^{1 / p}, \quad f \in L^{1} \cap L^{2},
\end{aligned}
$$

for $p, q \in(0, \infty)$ and non-negative weights $u$ and $v$. In the case $0<$ $p \leq 2=q$ our investigation yields a simple condition on weights $u$ and $v$ which is necessary and sufficient for (1.2) when $T$ is the Fourier transform. Taking $p=q=2$ and $u=v=\chi_{(0, z)}$ in (1.2) shows that (1.1) is included and, in view of the discussion below, may be interpreted as a weighted Lorentz norm inequality. To interpret (1.2) as a Lorentz space inequality we take $w(t)=t^{p-2} v(1 / t)$ and make the change of variable $t \rightarrow 1 / t$ in the right hand side of (1.2). It becomes

$$
\|T f\|_{\Lambda_{q}(u)} \leq C\|f\|_{\Gamma_{p}(w)}
$$

which expresses the boundedness of $T: \Gamma_{p}(w) \rightarrow \Lambda_{q}(u)$.

In [7], H. P. Heinig uses the Jodeit and Torchinsky result to greatly simplify the proofs of Fourier inequalities between weighted Lebesgue spaces, first proved in $[\mathbf{1}],[\mathbf{2}]$, and goes on to look at the Fourier transform as a map on $\Lambda_{p}(v)$. Our work here represents a development of his ideas in a different direction and would not have been possible without his cooperation and the inspiring discussions we were fortunate to have with him on the topic.

In the next section we introduce our tools and techniques; the level function, a class of averaging operators, and recent work on embeddings of the cone of quasi-concave functions. In Section 3 these are used to give conditions on $p, q, u$, and $v$ which are sufficient to imply (1.2). In Section 4 we focus on the Fourier transform, constructing the test functions that provide our necessary conditions. The last section shows that the conditions of Sections 3 and 4 reduce to a single simple condition in the case $0<p \leq 2=q$. 
To complete the introduction we present some notation used throughout the paper. The $n$-dimensional Fourier transform of $f$ is

$$
\mathcal{F} f(x)=\hat{f}(x)=\int_{\mathbf{R}^{n}} e^{-i x \cdot t} f(t) d t .
$$

We use the common notation $\hat{f}$ whenever possible, reserving $\mathcal{F}$ for situations where an operator name is required.

Let $L^{+}$be the set of Lebesgue measurable functions $h:(0, \infty) \rightarrow$ $[0, \infty]$ and, for $\alpha+\beta>0$, let $\Omega_{\alpha, \beta}$ denote the collection of those $h \in L^{+}$ such that $t^{\alpha} h(t)$ is non-decreasing and $t^{-\beta} h(t)$ is non-increasing. We only use two instances of this definition; the quasi-concave functions, $\Omega_{0,1}$; and a class that arises naturally in our situation, $\Omega_{2,0}$. We will use some distinguished elements of $\Omega_{2,0}$ : For each $z>0$ define $\omega_{z}$ by

$$
\omega_{z}(t)=\min \left(z^{-2}, t^{-2}\right) .
$$

The characteristic function of the set $E$ is denoted $\chi_{E}$. It takes the value 1 on $E$ and 0 otherwise. The notation $u_{n} \uparrow u$ means that $\left\{u_{n}\right\}$ is a non-decreasing sequence of functions that converge pointwise to $u$. On there other hand $\varphi \downarrow$ means that $\varphi$ is a non-increasing function.

We say that the two expressions $A$ and $B$ are equivalent and write $A \approx B$ when there are constants $m$ and $M$ such that $m A \leq B \leq M A$. The constants may depend on indices $p$ and $q$ but not on weights or functions. Similarly we write $A \lesssim B$ when there is a constant $M$ such that $A \leq M B$.

We take $[\mathbf{3}]$ as our standard reference for Banach Function Spaces, rearrangements and rearrangement invariant spaces. In particular we follow that text in our use of the Hardy-Littlewood-Pólya relation $h_{1} \prec h_{2}$ to mean

$$
\int_{0}^{t} h_{1}^{*} \leq \int_{0}^{t} h_{2}^{*}, \quad \text { for all } t>0 .
$$

\section{Preparation}

The level function construction of $[\mathbf{6}]$ and $[\mathbf{9}]$ has been developed further in $[\mathbf{1 2}],[\mathbf{1 3}],[\mathbf{1 4}]$. The properties we need here are contained in the next proposition which follows from [14, Proposition 1.2 and Proposition 5.1] by taking the measure $\lambda$ to be Lebesgue measure on $(0, \infty)$. 
Proposition 2.1. To each $h \in L^{+}$there corresponds a non-increasing function $h^{o} \in L^{+}$, called the level function of $h$, having the following properties:

a) For all non-increasing $\varphi \in L^{+}, \int_{0}^{\infty} \varphi h \leq \int_{0}^{\infty} \varphi h^{o}$.

b) If $0 \leq h_{n} \uparrow h$ pointwise then $h_{n}^{o} \uparrow h^{o}$ pointwise.

c) If $h$ is bounded and compactly supported then there exists a (necessarily finite or countable) collection of disjoint intervals $\left(a_{j}, b_{j}\right)$, each of finite length, such that

$$
h^{o}(x)=\frac{1}{b_{j}-a_{j}} \int_{a_{j}}^{b_{j}} h, \quad \text { for } a_{j} \leq x \leq b_{j},
$$

and $h^{o}(x)=h(x)$ for $x \notin \cup_{j}\left(a_{j}, b_{j}\right)$.

To use Proposition 2.1c) effectively we introduce the class $\mathcal{A}$ of averaging operators: Given a (necessarily finite or countable) collection $\left\{\left(a_{j}, b_{j}\right)\right\}$ of disjoint subintervals of $(0, \infty)$, each of finite length, we define the averaging operator $A$ by

$$
A h(x)= \begin{cases}\frac{1}{b_{j}-a_{j}} \int_{a_{j}}^{b_{j}} h & \text { for } a_{j} \leq x \leq b_{j} \\ h(x) & \text { otherwise. }\end{cases}
$$

The class $\mathcal{A}$ is the collection of all such operators $A$ and Proposition 2.1c) says that if $h$ is bounded and compactly supported then there exists an $A_{h} \in \mathcal{A}$ such that $h^{o}=A_{h} h$.

It is clear that for any $A \in \mathcal{A}$, if $h \in L^{+}$is non-increasing then so is $A h$. Moreover, [3, Proposition 2.3.7] shows that $A h \prec h$ so that if $h \in L^{+}$is non-increasing then $\int_{0}^{x} A h \leq \int_{0}^{x} h$ for all $x \geq 0$ and it follows that $\int_{0}^{\infty}(A h) \varphi \leq \int_{0}^{\infty} h \varphi$ whenever $\varphi \in L^{+}$is also non-increasing. As an illustration of the interplay between properties a) and c) of Proposition 2.1 we show that if $h \in L^{+}$is non-increasing then $h^{o}=h$. Taking $\varphi=\chi_{(0, x)}$ in Proposition 2.1a) we have

$$
\int_{0}^{x} h \leq \int_{0}^{x} h^{o}=\int_{0}^{x} A_{h} h \leq \int_{0}^{x} h
$$

for all $x>0$ and hence $h=h^{o}$. The other property of these averaging operators that we will use is their self-adjointness. An easy calculation shows that for any $A \in \mathcal{A}$

$$
\int_{0}^{\infty}(A h) \varphi=\int_{0}^{\infty} h(A \varphi), \quad h, \varphi \in L^{+} .
$$


It became evident in [14] that the effectiveness of level function techniques is not restricted to functions in Lebesgue spaces. The next lemma shows that it arises naturally in connection with the HardyLittlewood-Pólya relation, the fundamental relation in rearrangement invariant spaces. Recall that $h_{1} \prec h_{2}$ implies that $\left\|h_{1}\right\|_{X} \leq\left\|h_{2}\right\|_{X}$ for any rearrangement invariant space $X$. In particular if $h_{1} \prec h_{2}$ and $\varphi \in L^{+}$is non-increasing then $\int_{0}^{\infty} h_{1}^{*} \varphi \leq \int_{0}^{\infty} h_{2}^{*} \varphi$.

Lemma 2.2. Suppose that $h, u \in L^{+}$with $h$ non-increasing. Then

$$
\sup _{0 \leq \varphi \downarrow, \varphi \prec h} \int_{0}^{\infty} \varphi u=\sup _{A \in \mathcal{A}} \int_{0}^{\infty}(A h) u=\int_{0}^{\infty} h u^{o} .
$$

Proof: In view of the Monotone Convergence Theorem and Proposition $2.1 \mathrm{~b}$ ) it is enough to prove the lemma for $u$ bounded and of compact support. In this case there is an operator $A_{u} \in \mathcal{A}$ such that $A_{u} u=u^{o}$. We use the self-adjointness of $A_{u}$ to get

$$
\int_{0}^{\infty} h u^{o}=\int_{0}^{\infty} h\left(A_{u} u\right)=\int_{0}^{\infty}\left(A_{u} h\right) u \leq \sup _{A \in \mathcal{A}} \int_{0}^{\infty}(A h) u .
$$

Now $h$ is non-increasing so for any $A \in \mathcal{A}, A h$ is also non-increasing and we have $A h \prec h$. Thus

$$
\sup _{A \in \mathcal{A}} \int_{0}^{\infty}(A h) u \leq \sup _{0 \leq \varphi \downarrow, \varphi \prec h} \int_{0}^{\infty} \varphi u .
$$

For the remaining inequality we apply Proposition 2.1a) and then $\varphi \prec h$ to see that

$$
\sup _{0 \leq \varphi \downarrow, \varphi \prec h} \int_{0}^{\infty} \varphi u \leq \sup _{0 \leq \varphi \downarrow, \varphi \prec h} \int_{0}^{\infty} \varphi u^{o} \leq \int_{0}^{\infty} h u^{o} .
$$

The last inequality is valid because $u^{o}$ is non-increasing. This completes the proof.

The next two corollaries look at the extent to which this lemma carries over to norms.

Corollary 2.3. Suppose that $h \in L^{+}$is non-increasing and suppose that $X$ is a Banach Function Space of functions defined on $(0, \infty)$. Then

$$
\sup _{0 \leq \varphi \downarrow, \varphi \prec h}\|\varphi\|_{X}=\sup _{A \in \mathcal{A}}\|A h\|_{X} .
$$


Proof: Since $\varphi$ is non-negative, the norm in $X$ can be expressed as a suprema over all non-negative $u$ in the unit ball of the associate space $X^{\prime}$.

$$
\begin{aligned}
& \sup \left\{\|\varphi\|_{X}: 0 \leq \varphi \downarrow, \varphi \prec h\right\} \\
= & \sup \left\{\int_{0}^{\infty} \varphi u: 0 \leq \varphi \downarrow, \varphi \prec h, u \in L^{+},\|u\|_{X^{\prime}} \leq 1\right\} \\
= & \sup \left\{\int_{0}^{\infty}(A h) u: A \in \mathcal{A}, u \in L^{+},\|u\|_{X^{\prime}} \leq 1\right\} \\
= & \sup \left\{\|A h\|_{X}: A \in \mathcal{A}\right\} .
\end{aligned}
$$

Corollary 2.4. Suppose $1 \leq s<\infty$ and $h, u \in L^{+}$with $h$ non-increasing. Then

$$
\sup _{0 \leq \varphi \downarrow, \varphi \prec h}\|\varphi\|_{s, u}=\sup _{A \in \mathcal{A}}\|A h\|_{s, u} \leq\|h\|_{s, u^{o}} .
$$

Proof: Since $s>1, L_{u}^{s}$ is a Banach Function Space so the first statement follows from Corollary 2.3. By the Monotone Convergence Theorem it is enough to prove the second statement in the case that $h$ is bounded. Fix $A \in \mathcal{A}$. Since $h$ is non-increasing, so is $A h$. By Proposition 2.1a)

$$
\int_{0}^{\infty}(A h)^{s} u \leq \int_{0}^{\infty}(A h)^{s} u^{o} .
$$

We cut down $u^{o}$ to $u_{n}=\min \left(n, u^{o}\right)_{\chi_{[0, n)}}$ and note that $u_{n}$ is still nonincreasing. Since $h$ is bounded, so is $A h$ and thus $\int_{0}^{\infty}(A h)^{s} u_{n}<\infty$. Now $A h \prec h$ and $(A h)^{s-1} u_{n}$ is non-increasing so

$$
\int_{0}^{\infty}(A h)^{s} u_{n}=\int_{0}^{\infty} A h(A h)^{s-1} u_{n} \leq \int_{0}^{\infty} h(A h)^{s-1} u_{n} .
$$

This is the estimate we want in the case $s=1$. If $s>1$ we apply Hölder's inequality,

$$
\int_{0}^{\infty}(A h)^{s} u_{n} \leq\left(\int_{0}^{\infty} h^{s} u_{n}\right)^{1 / s}\left(\int_{0}^{\infty}(A h)^{s} u_{n}\right)^{1-1 / s},
$$

and divide by $\left(\int_{0}^{\infty}(A h)^{s} u_{n}\right)^{1-1 / s}$ to conclude that

$$
\left(\int_{0}^{\infty}(A h)^{s} u_{n}\right)^{1 / s} \leq\left(\int_{0}^{\infty} h^{s} u_{n}\right)^{1 / s}
$$

for all $s \geq 1$. Now let $n \rightarrow \infty$ to get

$$
\left(\int_{0}^{\infty}(A h)^{s} u^{o}\right)^{1 / s} \leq\left(\int_{0}^{\infty} h^{s} u^{o}\right)^{1 / s}
$$

which completes the proof. 
The next lemma will enable us to eliminate the level function when it appears in weight conditions.

Lemma 2.5. If $u \in L^{+}$then for all $x>0$

$$
\frac{1}{x} \int_{0}^{x} u^{o}=\sup _{A \in \mathcal{A}} \frac{1}{x} \int_{0}^{x} A u \leq 2 \sup _{y \geq x} \frac{1}{y} \int_{0}^{y} u \leq \frac{2}{x} \int_{0}^{x} u^{o} .
$$

Proof: Fix $x>0$. The function $\chi_{(0, x)}$ is non-increasing so the first statement follows from Lemma 2.2 and the self-adjointness of the operators $A \in \mathcal{A}$.

Now fix $A \in \mathcal{A}$ and let $\left(a_{j}, b_{j}\right)$ be the collection of disjoint intervals associated with $A$. If $x$ is not in any of the intervals $\left(a_{j}, b_{j}\right)$ then an easy calculation using the definition of $A$ shows that

$$
\int_{0}^{x} A u=\int_{0}^{x} u \text {. }
$$

For such an $x$ it is trivial that

$$
\frac{1}{x} \int_{0}^{x} A u \leq 2 \sup _{y \geq x} \frac{1}{y} \int_{0}^{y} u \text {. }
$$

Otherwise $x \in\left(a_{j}, b_{j}\right)$ for some $j$ and since $A u$ takes the value $\frac{1}{b_{j}-a_{j}} \int_{a_{j}}^{b_{j}} u$ on the interval $\left(a_{j}, b_{j}\right)$ we have

$$
\int_{0}^{x} A u=\int_{0}^{a_{j}} A u+\int_{a_{j}}^{x} A u=\int_{0}^{a_{j}} u+\frac{x-a_{j}}{b_{j}-a_{j}} \int_{a_{j}}^{b_{j}} u \leq \int_{0}^{x} u+\frac{x}{b_{j}} \int_{0}^{b_{j}} u .
$$

Note that $a_{j}<x<b_{j}$ implies $\frac{x-a_{j}}{b_{j}-a_{j}} \leq \frac{x}{b_{j}}$. It follow that for this $x$ we also have

$$
\frac{1}{x} \int_{0}^{x} A u \leq 2 \sup _{y \geq x} \frac{1}{y} \int_{0}^{y} u
$$

and, taking the supremum over all $A \in \mathcal{A}$ proves the middle inequality in (2.1). For the last inequality, Proposition 2.1a) and the monotonicity of $u^{o}$ show that for $y \geq x$,

$$
\frac{1}{y} \int_{0}^{y} u \leq \frac{1}{y} \int_{0}^{y} u^{o} \leq \frac{1}{x} \int_{0}^{x} u^{o}
$$

which completes the proof. 
The functions $\left(\int_{0}^{1 / t} f^{*}\right)^{2}$ that arise from the Jodeit and Torchinsky result (1.1) belong to the cone $\Omega_{2,0}$. That is to say, they are nonincreasing and become non-decreasing when multiplied by $t^{2}$. We employ recent results on weighted embeddings of this cone. The next proposition follows from [15, Theorems 2.6 and 2.7] with $\alpha=2$ and $\beta=0$. For related work on these embedding problems see [4], [5], [10] and [16].

Proposition 2.6. Suppose $p, q \in(0, \infty)$ and $u, v \in L^{+}$. Let

$$
B \equiv \sup _{h \in \Omega_{2,0}} \frac{\|h\|_{q / 2, u}}{\|h\|_{p / 2, v}} .
$$

If $p \leq q$ then

$B \approx \sup _{x>0}\left(\int_{0}^{\infty} \min \left(x^{-q}, t^{-q}\right) u(t) d t\right)^{2 / q}\left(\int_{0}^{\infty} \min \left(x^{-p}, t^{-p} v(t) d t\right)^{-2 / p}\right.$ and if $q<p$ then

$$
B \approx\left(\int_{0}^{\infty}\left(\frac{\int_{0}^{\infty} \min \left(x^{-q}, t^{-q}\right) u(t) d t}{\int_{0}^{\infty} \min \left(x^{-p}, t^{-p}\right) v(t) d t}\right)^{q /(p-q)} x^{-q} u(x) d x\right)^{2(p-q) /(p q)} .
$$

\section{Sufficient conditions}

In this section we give conditions on weights $u$ and $v$ and indices $p$ and $q$ which are sufficient to imply the inequality (1.2) for any operator $T$ of type $(1, \infty)$ and $(2,2)$. Since the Fourier transform is one such operator, the conditions imply weighted Fourier inequalities. Specifically, they ensure that the Fourier transform is bounded between certain weighted Lorentz spaces.

Theorem 3.1. Suppose that $0<p<\infty, 2 \leq q<\infty$ and $u, v \in L^{+}$. If $T$ is of type $(1, \infty)$ and $(2,2)$ then the inequality $(1.2)$ holds for all $f \in L^{1} \cap L^{2}$ with $C$ defined by

$$
C^{2}=D\left(\sup _{A \in \mathcal{A}, h \in \Omega_{2,0}} \frac{\|A h\|_{q / 2, u}}{\|h\|_{p / 2, v}}\right) .
$$

Here $D$ is a constant depending only on the operator $T$.

Proof: Since $T$ if of type $(1, \infty)$ and $(2,2),[8$, Theorem 4.6] shows that there exists a $D$ depending only on $T$ such that (1.1) holds. Fix $f \in$ $L^{1} \cap L^{2}$ and define $h_{f}$ and $\varphi_{f}$ by

$$
h_{f}(t)=\left(\int_{0}^{1 / t} f^{*}\right)^{2} \text { and } \varphi_{f}(x)=(T f)^{*}(x)^{2} / D .
$$


Clearly, $h_{f}(t)$ is non-increasing and we can see that $t^{2} h_{f}(t)$ is nondecreasing by recognizing it as (the square of) a moving average of the non-increasing function $f^{*}$. Thus $h_{f} \in \Omega_{2,0}$. The function $\varphi_{f}$ is also non-increasing and (1.1) shows that $\varphi_{f} \prec h_{f}$. We apply Corollary 2.3 with $X=L_{u}^{q / 2}$ to get

$$
\begin{aligned}
\frac{\left\|\varphi_{f}\right\|_{q / 2, u}}{\left\|h_{f}\right\|_{p / 2, v}} & \leq \sup _{0 \leq \varphi \downarrow, \varphi \prec h_{f}} \frac{\|\varphi\|_{q / 2, u}}{\left\|h_{f}\right\|_{p / 2, v}} \\
& =\sup _{A \in \mathcal{A}} \frac{\left\|A h_{f}\right\|_{q / 2, u}}{\left\|h_{f}\right\|_{p / 2, v}} \\
& \leq \sup _{A \in \mathcal{A}, h \in \Omega_{2,0}} \frac{\|A h\|_{q / 2, u}}{\|h\|_{p / 2, v}} .
\end{aligned}
$$

With $C$ as above this can be written

$$
\left\|\varphi_{f}\right\|_{q / 2, u} \leq C^{2} D^{-1}\left\|h_{f}\right\|_{p / 2, v} .
$$

Cancelling the $D$ 's and taking the square root of both sides yields

$$
\left(\int_{0}^{\infty}(T f)^{*}(x)^{q} u(x) d x\right)^{1 / q} \leq C\left(\int_{0}^{\infty}\left(\int_{0}^{1 / t} f^{*}\right)^{p} v(t) d t\right)^{1 / p}
$$

as required.

We can interpret this result as a sufficient condition for the boundedness of the operator $T$ between weighted Lorentz spaces.

Corollary 3.2. Suppose that $0<p<\infty, 2 \leq q<\infty$ and $u, w \in L^{+}$. Set $v(t)=t^{p-2} w(1 / t)$. If $T$ is of type $(1, \infty)$ and $(2,2)$ and if

$$
\sup _{A \in \mathcal{A}, h \in \Omega_{2,0}} \frac{\|A h\|_{q / 2, u}}{\|h\|_{p / 2, v}}<\infty
$$

then

$$
T: \Gamma_{p}(v) \rightarrow \Lambda_{q}(w) .
$$

The weight condition in Theorem 3.1 and Corollary 3.2 involves a supremum over all functions in $\Omega_{2,0}$. All that is required in the proof is the supremum over a smaller class, those functions of the form

$$
F(t)=\left(\int_{0}^{1 / t} f^{*}\right)^{2}, \quad f \in L^{+} .
$$


While each such $F$ is in $\Omega_{2,0}$, not every function in $\Omega_{2,0}$ is of the form (3.3). The next proposition shows that nothing is lost by working with the larger class.

Proposition 3.3. If $F \in \Omega_{2,0}$ then there is a non-decreasing sequence $\left\{F_{n}\right\}$ of functions of the form (3.3) such that

$$
F(t) \leq \lim _{n \rightarrow \infty} F_{n}(t) \leq 4 F(t), \quad t>0 .
$$

Proof: Fix $F \in \Omega_{2,0}$ and define $G$ by $G(x)=F(1 / x)^{1 / 2}$. It is easy to see that $G \in \Omega_{0,1}$. By $[\mathbf{1 5}$, Lemma 2.3] the least concave majorant $\tilde{G}$ of $G$ satisfies $G \leq \tilde{G} \leq 2 G$ and $\tilde{G}(t)$ is the limit of a non-decreasing sequence of functions of the form

$$
\int_{0}^{t} \int_{y}^{\infty} h_{n}(s) \frac{d s}{s} d y
$$

With $f_{n}(y)=f_{n}^{*}(y)=\int_{y}^{\infty} h_{n}(s) \frac{d s}{s}$ we have

$$
F(t)=G(1 / t)^{2} \leq \tilde{G}(1 / t)^{2}=\lim _{n \rightarrow \infty}\left(\int_{0}^{1 / t} f_{n}^{*}\right)^{2} \leq(2 G(1 / t))^{2}=4 F(t) .
$$

The condition (3.1) is not simple to verify. However, we can give a stronger condition in a form that is much easier to work with. The idea is to replace the weight $u$ by its level function to eliminate the supremum over all $A \in \mathcal{A}$.

Theorem 3.4. Suppose $0<p<\infty, 2 \leq q<\infty$ and $u, v \in L^{+}$. If $T$ is of type $(1, \infty)$ and $(2,2)$ then there exists a constant $C$ such that $(1.2)$ holds for all $f \in L^{1} \cap L^{2}$ provided either $p \leq q$ and

$$
\sup _{x \leq y}\left(\frac{x}{y} \int_{0}^{y} u\right)^{1 / q}\left(x^{p} \int_{x}^{\infty}\left(\frac{1}{t} \int_{0}^{t} v\right) \frac{d t}{t^{p}}\right)^{-1 / p}
$$

is finite, or $q<p, 1 / r=1 / q-1 / p$, and both

$$
\left(\int_{0}^{\infty} u\right)^{1 / q}\left(\int_{0}^{\infty} v\right)^{-1 / p}
$$

and

$\left(\int_{0}^{\infty}\left(\sup _{y \geq x} \frac{x}{y} \int_{0}^{y} u\right)^{r / q}\left(x^{p} \int_{x}^{\infty}\left(\frac{1}{t} \int_{0}^{t} v\right) \frac{d t}{t^{p}}\right)^{-r / q} x^{p} \int_{x}^{\infty} v(t) \frac{d t}{t^{p}} \frac{d x}{x}\right)^{1 / r}$

are finite. 
Proof: Applying Corollary 2.4 to the weight condition of Theorem 3.1 shows that if

$$
B^{2} \equiv \sup _{h \in \Omega_{2,0}} \frac{\|h\|_{q / 2, u^{o}}}{\|h\|_{p / 2, v}}<\infty
$$

then (1.2) holds. We use Proposition 2.6 with $u$ replaced by $u^{o}$. If $p \leq q$ then

$$
B \approx \sup _{x>0}\left(\int_{0}^{\infty} \min \left(x^{-q}, t^{-q}\right) u^{o}(t) d t\right)^{1 / q}\left(\int_{0}^{\infty} \min \left(x^{-p}, t^{-p}\right) v(t) d t\right)^{-1 / p} .
$$

The monotonicity of $u^{o}$ permits some simplification. We have

$$
\begin{aligned}
\int_{x}^{\infty} t^{-q} u^{o}(t) d t \leq u^{o}(x) \int_{x}^{\infty} & t^{-q} d t \\
& =\frac{x^{-q}}{q-1} u^{o}(x) \int_{0}^{x} d t \leq \frac{x^{-q}}{q-1} \int_{0}^{x} u^{o}(t) d t
\end{aligned}
$$

so

$$
\begin{aligned}
& \int_{0}^{\infty} \min \left(x^{-q}, t^{-q}\right) u^{o}(t) d t \\
& \quad=x^{-q} \int_{0}^{x} u^{o}(t) d t+\int_{x}^{\infty} t^{-q} u^{o}(t) d t \leq \frac{q x^{-q}}{q-1} \int_{0}^{x} u^{o}(t) d t .
\end{aligned}
$$

Combining this with Lemma 2.5 yields

$$
\int_{0}^{\infty} \min \left(x^{-q}, t^{-q}\right) u^{o}(t) d t \leq \frac{2 q x^{-q}}{q-1} \sup _{y \geq x} \frac{x}{y} \int_{0}^{y} u .
$$

Also, by an interchange of the order of integration,

$$
\int_{0}^{\infty} \min \left(x^{-p}, t^{-p}\right) v(t) d t=p \int_{x}^{\infty}\left(\frac{1}{t} \int_{0}^{t} v\right) \frac{d t}{t^{p}} .
$$

Now we have

$$
\begin{aligned}
B & \lesssim \sup _{x>0}\left(x^{-q} \sup _{y \geq x} \frac{x}{y} \int_{0}^{y} u\right)^{1 / q}\left(\int_{x}^{\infty}\left(\frac{1}{t} \int_{0}^{t} v\right) \frac{d t}{t^{p}}\right)^{-1 / p} \\
& =\sup _{x \leq y}\left(\frac{x}{y} \int_{0}^{y} u\right)^{1 / q}\left(x^{p} \int_{x}^{\infty}\left(\frac{1}{t} \int_{0}^{t} v\right) \frac{d t}{t^{p}}\right)^{-1 / p}
\end{aligned}
$$

This completes the proof in the case $p \leq q$. 
If $q<p$ then Proposition 2.6 shows that

$$
B \approx\left(\int_{0}^{\infty}\left(\frac{\int_{0}^{\infty} \min \left(x^{-q}, t^{-q}\right) u^{o}(t) d t}{\int_{0}^{\infty} \min \left(x^{-p}, t^{-p}\right) v(t) d t}\right)^{r / p} x^{-q} u^{o}(x) d x\right)^{1 / r}
$$

and we use the monotonicity of $u^{o}$ as above to get

$$
B \lesssim\left(\int_{0}^{\infty}\left(\frac{x^{-q} \int_{0}^{x} u^{o}(t) d t}{\int_{0}^{\infty} \min \left(x^{-p}, t^{-p}\right) v(t) d t}\right)^{r / p} x^{-q} u^{o}(x) d x\right)^{1 / r}
$$

We omit the details of the integration by parts that reduces this last expression to

$$
\begin{aligned}
\left(q \int_{0}^{\infty}\left(\int_{0}^{x} u^{o}(t) d t\right)^{r / q}\right. & \left(x^{p} \int_{0}^{\infty} \min \left(x^{-p}, t^{-p}\right) v(t) d t\right)^{-r / q} x^{p} \int_{x}^{\infty} v(t) \frac{d t}{t^{p}} \frac{d x}{x} \\
+ & \left.\frac{q}{r}\left(\int_{0}^{\infty} u^{o}(t) d t\right)^{r / q}\left(\int_{0}^{\infty} v(t) d t\right)^{-r / p}\right)^{1 / r}
\end{aligned}
$$

Now Lemma 2.5 and (3.5), together with the fact that $\int_{0}^{\infty} u^{o}=\int_{o}^{\infty} u$ give us

$$
\begin{array}{r}
B \lesssim\left(\int_{0}^{\infty}\left(\sup _{y \geq x} \frac{x}{y} \int_{0}^{x} u\right)^{r / q}\left(x^{p} \int_{x}^{\infty}\left(\frac{1}{t} \int_{0}^{t} v\right) \frac{d t}{t^{p}}\right)^{-r / q} x^{p} \int_{x}^{\infty} v(t) \frac{d t}{t^{p}} \frac{d x}{x}\right. \\
\left.+\left(\int_{0}^{\infty} u\right)^{r / q}\left(\int_{0}^{\infty} v\right)^{-r / p}\right)^{1 / r}
\end{array}
$$

This completes the proof.

Another way to replace (3.1) by a stronger sufficient condition that does not involve a supremum over all $A \in \mathcal{A}$ is to note that for any $A \in \mathcal{A}$ and any non-increasing $h$,

$$
A h(x) \leq \frac{1}{x} \int_{0}^{x} A h \leq \frac{1}{x} \int_{0}^{x} h=h^{* *}(x) .
$$

Thus

$$
\sup _{A \in \mathcal{A}, h \in \Omega_{2,0}} \frac{\|A h\|_{q / 2, u}}{\|h\|_{p / 2, v}} \leq \sup _{h \in \Omega_{2,0}} \frac{\left\|h^{* *}\right\|_{q / 2, u}}{\|h\|_{p / 2, v}} .
$$

It is possible to write down an expression equivalent to this last supremum by following the method of [15, Theorem 5.1]. However, we do not present the result here because there are many weight conditions involved and the result, while sufficient for (1.2), is unlikely to be necessary. 


\section{Necessary conditions}

The results of this section build up to the construction of functions that will serve as a test functions in (1.2). Ideally, we want to construct a small collection of functions $f$ with the property that if (1.2) holds for these $f$ then the sufficient condition of Theorem 3.4 also holds. The extent to which we succeed is seen in Theorem 5.1.

The sufficient condition of Theorem 3.1 is a supremum over all $A \in \mathcal{A}$ and all $h \in \Omega_{2,0}$ but the test functions we construct will be indexed by $A \in \mathcal{A}$ and only certain functions in $\Omega_{2,0}$. Since

$$
\omega_{z}(x)=\min \left(z^{-2}, t^{-2}\right)=\left(\int_{0}^{1 / t} \chi_{(0,1 / z)}\right)^{2}
$$

we see that $\omega_{z} \in \Omega_{2,0}$ for each $z>0$. Moreover this representation of $\omega_{z}$ suggests that we should look at functions $f$ whose rearrangement is $\chi_{(0,1 / z)}$.

Even for a fixed $z>0$, there is a large class of functions $f$ equimeasurable with $\chi_{(0,1 / z)}$. Our task is to show that for each $A \in \mathcal{A}$ there is one such $f$ whose Fourier transform satisfies $(\tilde{f})^{*^{2}} \geq A \omega_{z}$. This is done, up to a small epsilon and a constant multiple, in Theorem 4.6. Our necessary condition for (1.2) is given in Corollary 4.8.

We begin with an estimate of the rearrangement of a function that arises as the Fourier transform of a characteristic function.

Lemma 4.1. Let $S(x)=\sin (x) / x$. Then $S^{*}(y) \geq(3 \pi+y)^{-1}$.

Proof: We estimate the distribution function $\mu_{S}(\alpha)$ of $S$ as follows. For $\alpha>0$,

$$
\begin{aligned}
\mu_{S}(\alpha) & \equiv|\{x:|\sin (x) / x|>\alpha\}| \\
& =2|\{x>0:|\sin (x)|>x \alpha\}| \\
& =2 \sum_{n=1}^{\infty}|\{x \in((n-1) \pi, n \pi):|\sin (x)|>x \alpha\}| .
\end{aligned}
$$


For $x \in((n-1) \pi, n \pi)$ the condition $|\sin (x)|>x \alpha$ is weaker than the condition $|\sin (x)|>n \pi \alpha$ so we have

$$
\begin{aligned}
\mu_{S}(\alpha) & \geq 2 \sum_{n=1}^{\infty}|\{x \in((n-1) \pi, n \pi):|\sin (x)|>n \pi \alpha\}| \\
& =2 \sum_{n=1}^{\infty}|\{x \in(0, \pi): \sin (x)>n \pi \alpha\}| \\
& =4 \sum_{n=1}^{\infty}|\{x \in(0, \pi / 2): \sin (x)>n \pi \alpha\}|
\end{aligned}
$$

using the symmetry of $\sin (x)$. Since the condition $\sin (x)>n \pi \alpha$ is never satisfied for $n \pi \alpha>1$ we may restrict the sum to those $n$ for which $n \pi \alpha \leq 1$. To this end we let $N$ be the integer satisfying $(1 /(\pi \alpha))-1<$ $N \leq 1 /(\pi \alpha)$. Also, $\sin (x) \geq 2 x / \pi$ for $0 \leq x \leq \pi / 2$ so we have

$$
\begin{aligned}
\mu_{S}(\alpha) & \geq 4 \sum_{n=1}^{N}|\{x \in(0, \pi / 2): \sin (x)>n \pi \alpha\}| \\
& \geq 4 \sum_{n=1}^{N}|\{x \in(0, \pi / 2): 2 x / \pi>n \pi \alpha\}| \\
& =4 \sum_{n=1}^{N}\left(\pi / 2-n \pi^{2} \alpha / 2\right) \\
& =2 \pi(N-\pi \alpha N(N+1) / 2) .
\end{aligned}
$$

The definition of $N$ completes the estimate of $\mu_{S}(\alpha)$.

$$
\mu_{S}(\alpha) \geq 2 \pi\left(\frac{1}{\pi \alpha}-1-\frac{\pi \alpha}{2} \frac{1}{\pi \alpha}\left(\frac{1}{\pi \alpha}+1\right)\right)=\frac{1}{\alpha}-3 \pi .
$$

Now for any $y>0$,

$$
S^{*}(y)=\inf \left\{\alpha: \mu_{S}(\alpha) \leq y\right\} \geq \inf \{\alpha:(1 / \alpha)-3 \pi \leq y\}=(3 \pi+y)^{-1} .
$$

Corollary 4.2. If $z>0$ and $f=\chi_{(0,1 / z)}$ then $(\hat{f})^{*}(y) \geq(3 \pi z+y / 2)^{-1}$.

Proof: The (one-dimensional) Fourier transform of $f$ is

$$
\hat{f}(x)=\int_{0}^{1 / z} e^{-i x t} d t=\frac{e^{-i x / z}-1}{-i x} .
$$


If $S(x)=\sin (x) / x$ as in Lemma 4.1 we have

$$
\begin{aligned}
|\hat{f}(x)| & =\left|\frac{1}{z} \frac{e^{-i x /(2 z)}}{x /(2 z)} \frac{e^{i x /(2 z)}-e^{-i x /(2 z)}}{2 i}\right| \\
& =\frac{1}{z}\left|\frac{\sin (x /(2 z))}{x /(2 z)}\right| \\
& =\frac{1}{z}|S(x /(2 z))| .
\end{aligned}
$$

The rearrangement satisfies $(a g)^{*}=|a| g^{*}$ for any complex number $a$ and function $g$. Also, since we are taking the rearrangement with respect to Lebesgue measure, it respects dilation. That is, if $g_{a}(x)=g(a x)$ then $g_{a}^{*}(y)=g^{*}(|a| y)$. These properties, together with Lemma 4.1 show that

$$
(\hat{f})^{*}(y)=\frac{1}{z} S^{*}(y /(2 z)) \geq \frac{1}{z} \frac{1}{3 \pi+(y /(2 z))}=\frac{1}{3 \pi z+y / 2} .
$$

The Fourier transform of a dilation of $f$ is the inverse dilation of the Fourier transform of $f$, up to a scaling factor. The next result and its corollary are counter-intuitive because the same dilation appears in both the time and frequency domains. To achieve this we break up $f$ into several equimeasurable pieces with disjoint supports. Since we are only concerned with rearrangements we are free to multiply each piece by high frequency functions of unit modulus to produce translation in the frequency domain and prevent the Fourier transforms of the pieces from reinforcing.

Lemma 4.3. Suppose that $f: \mathbf{R} \rightarrow \mathbf{C}$ is a compactly supported $L^{1}$ function and $k$ is a positive integer. For any $\varepsilon>0$ there exists a compactly supported $L^{1}$ function $g$ such that $g^{*}(s)=f^{*}(s / k)$ for $s>0$ and

$$
(\hat{f})^{*}(y / k)-\varepsilon \leq(\hat{g})^{*}(y) \leq(\hat{f})^{*}(y / k)+\varepsilon
$$

for $y>0$.

Proof: We show that for $T$ and $X$ sufficiently large,

$$
g(t)=\sum_{j=1}^{k} e^{i j X t} f(t+j T)
$$

will do. It is clear that such a $g$ is compactly supported and in $L^{1}$. Choose $T$ so large that the supports of $f(t+j T), j=1, \ldots, k$ are 
disjoint. Then no matter what $X$ is, for all $\alpha>0$ we have

$$
|\{t:|g(t)|>\alpha\}|=\sum_{j=1}^{k}|\{t:|f(t+j T)|>\alpha\}|=k|\{t:|f(t)|>\alpha\}|
$$

using the translation invariance of Lebesgue measure. We use this to express the rearrangement of $g$ in terms of the rearrangement of $f$.

$$
\begin{aligned}
g^{*}(s) & =\inf \{\alpha:|\{t:|g(t)|>\alpha\}| \leq s\} \\
& =\inf \{\alpha:|\{t:|f(t)|>\alpha\}| \leq s / k\}=f^{*}(s / k) .
\end{aligned}
$$

Now we turn to the Fourier transform of $g$ and the choice of $X$. By the Riemann-Lebesgue Lemma we have

$$
\lim _{|x| \rightarrow \infty}|\hat{f}(x)|=0
$$

so we may choose $X$ so large that $|\hat{f}(x)|<\varepsilon / k$ whenever $|x| \geq X / 2$. Since

$$
\hat{g}(x)=\sum_{j=1}^{k} e^{i(x-j X) j T} \hat{f}(x-j X)
$$

we see that if $x \in(j X-X / 2, j X+X / 2)$ for some $j$ then only the $j$ th term of the sum can contribute more than $\varepsilon / k$ so

$$
|\hat{f}(x-j X)|-\varepsilon \leq|\hat{g}(x)| \leq|\hat{f}(x-j X)|+\varepsilon
$$

and if $x \notin(j X-X / 2, j X+X / 2)$ for any $j$ then none of the terms in the sum can contribute more than $\varepsilon / k$ so $|\hat{g}(x)|<\varepsilon$. Thus, for $\alpha>\varepsilon$ we have

$$
\begin{aligned}
|\{x:|\hat{g}(x)|>\alpha\}| & =\sum_{j=1}^{k}|\{x \in(j X-X / 2, j X+X / 2):|\hat{g}(x)|>\alpha\}| \\
& \leq \sum_{j=1}^{k}|\{x \in(j X-X / 2, j X+X / 2):|\hat{f}(x-j X)|>\alpha-\varepsilon\}| \\
& =\sum_{j=1}^{k}|\{x \in(-X / 2, X / 2):|\hat{f}(x)|>\alpha-\varepsilon\}| \\
& \leq k|\{x:|\hat{f}(x)|>\alpha-\varepsilon\}| .
\end{aligned}
$$


This implies that if $(\hat{g})^{*}(y)>\varepsilon$ then

$$
\begin{aligned}
(\hat{g})^{*}(y) & =\inf \{\alpha:|\{x:|\hat{g}(x)|>\alpha\}| \leq y\} \\
& \leq \inf \{\alpha:|\{x:|\hat{f}(x)|>\alpha-\varepsilon\}| \leq y / k\} \\
& =\inf \{\alpha-\varepsilon:|\{x:|\hat{f}(x)|>\alpha-\varepsilon\}| \leq y / k\}+\varepsilon \\
& =(\hat{f})^{*}(y / k)+\varepsilon .
\end{aligned}
$$

Of course, if $(\hat{g})^{*}(y) \leq \varepsilon$ then we also have $(\hat{g})^{*}(y) \leq(\hat{f})^{*}(y / k)+\varepsilon$ so we have established the second inequality in (4.1).

To prove the first inequality in (4.1) we observe that for all $\alpha>0$, (4.2) implies that

$$
\begin{aligned}
|\{x:|\hat{g}(x)|>\alpha\}| & \geq \sum_{j=1}^{k}|\{x \in(j X-X / 2, j X+X / 2):|\hat{g}(x)|>\alpha\}| \\
& \geq \sum_{j=1}^{k}|\{x \in(j X-X / 2, j X+X / 2):|\hat{f}(x-j X)|>\alpha+\varepsilon\}| \\
& =\sum_{j=1}^{k}|\{x \in(-X / 2, X / 2):|\hat{f}(x)|>\alpha+\varepsilon\}| \\
& =k|\{x:|\hat{f}(x)|>\alpha+\varepsilon\}|
\end{aligned}
$$

where the last equality uses the fact that $|\hat{f}(x)|<\varepsilon$ for $|x| \geq X / 2$. Now

$$
\begin{aligned}
(\hat{g})^{*}(y) & =\inf \{\alpha:|\{x:|\hat{g}(x)|>\alpha\}| \leq y\} \\
& \geq \inf \{\alpha:|\{x:|\hat{f}(x)|>\alpha+\varepsilon\}| \leq y / k\} \\
& =\inf \{\alpha+\varepsilon:|\{x:|\hat{f}(x)|>\alpha+\varepsilon\}| \leq y / k\}-\varepsilon \\
& \geq \inf \{\alpha:|\{x:|\hat{f}(x)|>\alpha\}| \leq y / k\}-\varepsilon \\
& =(\hat{f})^{*}(y / k)-\varepsilon
\end{aligned}
$$

as required. This completes the proof.

Corollary 4.4. Given $z>0, r>0$ and $\varepsilon>0$ there exists a compactly supported $L^{1}$ function $g: \mathbf{R} \rightarrow \mathbf{C}$ such that

$$
g^{*}=\chi_{[0,1 / z)} \quad \text { and } \quad(\hat{g})^{*}(y)+\varepsilon \geq(3 \pi(r+1) z+y /(2 r))^{-1} .
$$


Proof: Let $k$ be the positive integer that satisfies $k-1<r \leq k$ and set $f=\chi_{(0,1 /(k z))}$. Choose $g$ by Lemma 4.3 so that

$$
g^{*}(s)=f^{*}(s / k)=\chi_{[0,1 /(k z))}(s / k)=\chi_{[0,1 / z)}(s)
$$

and

$(\hat{g})^{*}(y) \geq(\hat{f})^{*}(y / k)-\varepsilon \geq(3 \pi k z+y /(2 k))^{-1}-\varepsilon \geq(3 \pi(r+1) z+y /(2 r))^{-1}-\varepsilon$.

Here we have used Corollary 4.2 to estimate $(\hat{f})^{*}$.

The same idea used in Lemma 4.3 is used below to keep the Fourier transforms of the terms of a sum from reinforcing in the frequency domain. This time the summands are not equimeasurable, however, so the estimate is rather coarse.

Lemma 4.5. Suppose that $\left\{f_{j}\right\}$ is a sequence of compactly supported functions mapping $\mathbf{R}$ to $\mathbf{C}$ and satisfying $f_{j}^{*}=\chi_{\left[0, s_{j}\right)}$ for $j=1,2, \ldots$ with $\sum_{j=1}^{\infty} s_{j} \equiv s_{0}<\infty$. Then for any $\varepsilon>0$ there exists a $g$ such that $g^{*}=\chi_{\left[0, s_{0}\right)}$ and

$$
(\hat{g})^{*}(y) \geq\left(\hat{f}_{j}\right)^{*}(y)-\varepsilon, \quad y>0 ; j=1,2, \ldots .
$$

Proof: We show that there are sequences $\left\{T_{j}\right\}$ and $\left\{X_{j}\right\}$ so that

$$
g(t)=\sum_{j=1}^{\infty} e^{i X_{j} t} f_{j}\left(t+T_{j}\right)
$$

will do. Let $T_{1}=0$ and suppose that $T_{1}, \ldots, T_{n-1}$ have been chosen. Choose $T_{n}$ so large that the supports of $f_{j}\left(t+T_{j}\right), j=1, \ldots, n$ are disjoint. Then no matter what the sequence $\left\{X_{j}\right\}$ is, for all $\alpha>0$ we have

$$
|\{t:|g(t)|>\alpha\}|=\sum_{j=1}^{\infty}\left|\left\{t:\left|f_{j}\left(t+T_{j}\right)\right|>\alpha\right\}\right| .
$$

Since $f_{j}^{*}=\chi_{\left[0, s_{j}\right)}$ this sum is zero for $\alpha \geq 1$ and takes the value $s_{0}$ for $\alpha<1$. It follows that $g^{*}=\chi_{\left[0, s_{0}\right)}$.

To construct the sequence $\left\{X_{j}\right\}$ we first apply the Riemann-Lebesgue Lemma to each $\hat{f}_{j}$ to select an $R_{j}>0$ such that $\left|\hat{f}_{j}(x)\right|<\varepsilon 2^{-j}$ whenever $|x| \geq R_{j}$. Now set $X_{1}=0$ and suppose that $X_{1}, \ldots, X_{n-1}$ have been chosen. Choose $X_{n}$ so large that no two of the intervals $\left(X_{j}-R_{j}, X_{j}+\right.$ $\left.R_{j}\right), j=1, \ldots, n$ intersect. 
Since the sum that defines $g$ converges in $L^{1}$, the sum

$$
\hat{g}(x)=\sum_{j=1}^{\infty} e^{i\left(x-X_{j}\right) T_{j}} \hat{f}_{j}\left(x-X_{j}\right)
$$

converges in $L^{\infty}$. It follows that for almost every $x \in\left(X_{j}-R_{j}, X_{j}+R_{j}\right)$,

$$
\left|\hat{f}_{j}\left(x-X_{j}\right)\right|-\varepsilon \leq|\hat{g}(x)|
$$

since the $\ell$ th term of the sum contributes at most $\varepsilon 2^{-\ell}$ unless $\ell=j$. For $\alpha>0$ we have

$$
\begin{aligned}
|\{x:|\hat{g}(x)|>\alpha\}| & \geq \sum_{j=1}^{\infty}\left|\left\{x \in\left(X_{j}-R_{j}, X_{j}+R_{j}\right):|\hat{g}(x)|>\alpha\right\}\right| \\
& \geq \sum_{j=1}^{\infty}\left|\left\{x \in\left(X_{j}-R_{j}, X_{j}+R_{j}\right):\left|\hat{f}_{j}\left(x-X_{j}\right)\right|>\alpha+\varepsilon\right\}\right| \\
& =\sum_{j=1}^{\infty}\left|\left\{x \in\left(-R_{j}, R_{j}\right):\left|\hat{f}_{j}(x)\right|>\alpha+\varepsilon\right\}\right| \\
& =\sum_{j=1}^{\infty}\left|\left\{x:\left|\hat{f}_{j}(x)\right|>\alpha+\varepsilon\right\}\right| \\
& \geq \sup _{j \geq 1}\left|\left\{x:\left|\hat{f}_{j}(x)\right|>\alpha+\varepsilon\right\}\right| .
\end{aligned}
$$

Here we have used the fact that for $x \notin\left(-R_{j}, R_{j}\right),\left|\hat{f}_{j}(x)\right|<\varepsilon 2^{-j}<\varepsilon<$ $\alpha+\varepsilon$. Thus, for each $j$,

$$
\begin{aligned}
(\hat{g})^{*}(y) & =\inf \{\alpha:|\{x:|\hat{g}(x)|>\alpha\}| \leq y\} \\
& \geq \inf \left\{\alpha:\left|\left\{x:\left|\hat{f}_{j}(x)\right|>\alpha+\varepsilon\right\}\right| \leq y\right\} \\
& =\inf \left\{\alpha+\varepsilon:\left|\left\{x:\left|\hat{f}_{j}(x)\right|>\alpha+\varepsilon\right\}\right| \leq y\right\}-\varepsilon \\
& \geq \inf \left\{\alpha:\left|\left\{x:\left|\hat{f}_{j}(x)\right|>\alpha\right\}\right| \leq y\right\}-\varepsilon \\
& =\left(\hat{f}_{j}\right)^{*}(y)-\varepsilon .
\end{aligned}
$$

This completes the proof. 
Now we are ready to put these results together to construct a test function $f$ for each averaging operator $A \in \mathcal{A}$ and $z>0$. Recall that $\omega_{z}(t)=\min \left(z^{-2}, t^{-2}\right)$.

Theorem 4.6. Suppose $z>0$ and $A \in \mathcal{A}$. For each $\varepsilon>0$ there exists a function $f: \mathbf{R} \rightarrow \mathbf{C}$ with $f^{*} \leq \chi_{[0,1 / z)}$ and

$$
\left(A \omega_{z}\right)^{1 / 2} \leq c_{1}\left((\hat{f})^{*}+\varepsilon\right)
$$

with $c_{1}=87.7$.

Proof: Fix $z>0$ and $A \in \mathcal{A}$. The intervals associated with $A$ are disjoint so there exists at most one that constains $z$. Let $\left(a_{0}, b_{0}\right)$ be the interval of $A$ containing $z$ if it exists. Otherwise let $a_{0}=b_{0}=z$. Of the remaining intervals of $A$ we select those that lie to the right of $z$ and are long compared to their distance from zero. Set

$$
J=\left\{j: z \leq a_{j} \leq b_{j} / 2\right\} .
$$

Let $f_{0}=\chi_{(0,1 /(4 z))}$. By Corollary 4.2 we have

$$
f_{0}^{*}=\chi_{(0,1 /(4 z))} \text { and }\left(\hat{f}_{0}\right)^{*}(y) \geq(12 \pi z+y / 2)^{-1} .
$$

Choose $g_{0}$ by Corollary 4.4 with $z$ replaced by $4 z$ and $r=r_{0} \equiv$ $\sqrt{b_{0} /(24 \pi z)}$ so that $g_{0}^{*}=\chi_{(0,1 /(4 z))}$ and

$\left(\hat{g}_{0}\right)^{*}(y)+\varepsilon / 2 \geq\left(12 \pi\left(r_{0}+1\right) z+y /\left(2 r_{0}\right)\right)^{-1}=\left(\left(1+y / b_{0}\right) \sqrt{6 \pi b_{0} z}+12 \pi z\right)^{-1}$.

For each $j \in J$ we choose $g_{j}$ by Corollary 4.4 with $z$ replaced by $4\left(z+a_{j}\right)$ and $r=r_{j} \equiv \sqrt{b_{j} /\left(24 \pi\left(z+a_{j}\right)\right)}$ so that $g_{j}^{*}=\chi_{\left(0,1 /\left(4\left(z+a_{j}\right)\right)\right)}$ and

$$
\begin{aligned}
\left(\hat{g}_{0}\right)^{*}(y)+\varepsilon / 2 & \geq\left(12 \pi\left(r_{j}+1\right)\left(z+a_{j}\right)+y /\left(2 r_{j}\right)\right)^{-1} \\
& \geq\left(\left(1+y / b_{j}\right) \sqrt{6 \pi b_{j}\left(z+a_{j}\right)}+12 \pi\left(z+a_{j}\right)\right)^{-1} .
\end{aligned}
$$

We plan to apply Lemma 4.5 to the collection $\left\{f_{0}, g_{0}, g_{j}: j \in J\right\}$. To do this we must show that the sum

$$
s_{0} \equiv \frac{1}{4 z}+\frac{1}{4 z}+\sum_{j \in J} \frac{1}{4\left(z+a_{j}\right)}
$$

converges. The definition of $J$ shows that $a_{j} \in(z, \infty)$ for all $j \in J$. Moreover, since the intervals indexed by $J$ satisfy $b_{j}>2 a_{j}$, at most 
one $a_{j}$ can be in any interval of the form $\left(2^{m} z, 2^{m+1} z\right)$ for $m=0,1, \ldots$. Thus

$$
s_{0} \leq \frac{1}{4 z}+\frac{1}{4 z}+\sum_{m=0}^{\infty} \frac{1}{4\left(z+2^{m} z\right)} \leq \frac{1}{2 z}+\sum_{m=0}^{\infty} \frac{2^{-2-m}}{z}=\frac{1}{2 z}+\frac{1}{2 z}=\frac{1}{z}<\infty .
$$

By Lemma 4.5 there is a function $f$ such that $f^{*}=\chi_{\left[0, s_{0}\right)} \leq \chi_{[0,1 / z)}$ which satisfies

$$
\begin{aligned}
& (\hat{f})^{*}(y) \geq\left(\hat{f}_{0}\right)^{*}(y)-\varepsilon / 2, \\
& (\hat{f})^{*}(y) \geq\left(\hat{g}_{0}\right)^{*}(y)-\varepsilon / 2, \\
& (\hat{f})^{*}(y) \geq\left(\hat{g}_{j}\right)^{*}(y)-\varepsilon / 2, \quad j \in J .
\end{aligned}
$$

Using the above estimates of $\left(\hat{f}_{0}\right)^{*},\left(\hat{g}_{0}\right)^{*}$, and $\left(\hat{g}_{j}\right)^{*}$, we get

$$
\begin{aligned}
& (\hat{f})^{*}(y)+\varepsilon \geq(12 \pi z+y / 2)^{-1} \\
& (\hat{f})^{*}(y)+\varepsilon \geq\left(\left(1+y / b_{0}\right) \sqrt{6 \pi b_{0} z}+12 \pi z\right)^{-1}, \quad \text { and } \\
& (\hat{f})^{*}(y)+\varepsilon \geq\left(\left(1+y / b_{j}\right) \sqrt{6 \pi b_{j}\left(z+a_{j}\right)}+12 \pi\left(z+a_{j}\right)\right)^{-1}, \quad j \in J
\end{aligned}
$$

To complete the proof we must show that $A \omega_{z}(y)^{1 / 2} \leq c_{1}\left((\hat{f})^{*}(y)+\varepsilon\right)$. We do this in three cases.

Case 1: $A \omega_{z}(y) \leq 2 \omega_{z}(y)$. We have

$$
A \omega_{z}(y)^{1 / 2} \leq \sqrt{2} \min (1 / z, 1 / y) \leq \sqrt{2}\left(\frac{12 \pi+1 / 2}{12 \pi z+y / 2}\right) .
$$

Now (4.4) yields

$$
A \omega_{z}(y)^{1 / 2} \leq \sqrt{2}(12 \pi+1 / 2)\left((\hat{f})^{*}(y)+\varepsilon\right) \leq c_{1}\left((\hat{f})^{*}(y)+\varepsilon\right) .
$$

Case 2: $a_{0}<y<b_{0}$. This case only arises when $\left(a_{0}, b_{0}\right)$ is an interval of $A$ containing $z$. Since $\omega_{z}$ is non-increasing,

$$
A \omega_{z}(y)=\frac{1}{b_{0}-a_{0}} \int_{a_{0}}^{b_{0}} \omega_{z} \leq \frac{1}{b_{0}} \int_{0}^{b_{0}} \omega_{z} \leq \frac{1}{b_{0}} \int_{0}^{\infty} \omega_{z}=\frac{2}{b_{0} z} .
$$

Since $y<b_{0}$ and $z<b_{0}$ we have

$$
A \omega_{z}(y)^{1 / 2}=\frac{\sqrt{2}}{\sqrt{b_{0} z}}=\frac{\sqrt{2}(2 \sqrt{6 \pi}+12 \pi)}{2 \sqrt{6 \pi} \sqrt{b_{0} z}+12 \pi \sqrt{b_{0} z}} \leq \frac{\sqrt{2}(2 \sqrt{6 \pi}+12 \pi)}{\left(1+y / b_{0}\right) \sqrt{6 \pi b_{0} z}+12 \pi z}
$$


and we may use (4.5) to get

$$
A \omega_{z}(y)^{1 / 2} \leq \sqrt{2}(2 \sqrt{6 \pi}+12 \pi)\left((\hat{f})^{*}(y)+\varepsilon\right) \leq c_{1}\left((\hat{f})^{*}(y)+\varepsilon\right) .
$$

Case 3: $A \omega_{z}(y)>2 \omega_{z}(y)$ and $y \notin\left(a_{0}, b_{0}\right)$. Since $A \omega_{z}$ is not equal to $\omega_{z}$ at $y$ it follows that $y$ must be in some interval $(a, b)$ of $A$. We have specifically excluded the possibility that $a<z<b$. If $a<y<b \leq z$ then $A \omega_{z}$ is the average of the constant function $z^{-2}$ on $(a, b)$ so $A \omega_{z}(y)=$ $z^{-2}=\omega_{z}(y)$ which rules out this possibility as well. Therefore, this case only arises when $z \leq a<y<b$ for some interval $(a, b)$ of $A$. We can use this information to calculate that

$$
\omega_{z}(y)=y^{-2} \text { and } A \omega_{z}(y)=1 /(a b) .
$$

Now

$$
a=\frac{1}{A \omega_{z}(y) b}<\frac{1}{2 \omega_{z}(y) b}=\frac{y^{2}}{2 b} \leq \frac{b}{2}
$$

so the interval $(a, b)$ is $\left(a_{j}, b_{j}\right)$ for some $j \in J$. For this $j$ we have $2 \sqrt{a_{j} b_{j}} \geq 2 a_{j} \geq z+a_{j}$ so

$$
\begin{aligned}
A \omega_{z}(y)^{1 / 2}=\frac{1}{\sqrt{a_{j} b_{j}}} & =\frac{2 \sqrt{12 \pi}+24 \pi}{2 \sqrt{6 \pi\left(2 a_{j}\right) b_{j}}+12 \pi\left(2 \sqrt{a_{j} b_{j}}\right)} \\
& \leq \frac{2 \sqrt{12 \pi}+24 \pi}{\left(1+y / b_{j}\right) \sqrt{6 \pi\left(z+a_{j}\right) b_{j}}+12 \pi\left(z+a_{j}\right)} \\
& \leq(2 \sqrt{12 \pi}+24 \pi)\left((\hat{f})^{*}(y)+\varepsilon\right) \leq c_{1}\left((\hat{f})^{*}(y)+\varepsilon\right) .
\end{aligned}
$$

Here we have applied (4.6). This completes the proof.

It is a simple matter to extend this result to functions on $\mathbf{R}^{n}$.

Corollary 4.7. Suppose $n$ is a positive integer, $z>0$ and $A \in \mathcal{A}$. For each $\varepsilon>0$ there exists a function $f: \mathbf{R}^{n} \rightarrow \mathbf{C}$ with $f^{*} \leq \chi_{[0,1 / z)}$ and

$$
\left(A \omega_{z}\right)^{1 / 2} \leq c_{n}\left((\hat{f})^{*}+\varepsilon\right)
$$

with $c_{n}=87.7(3 \pi+1 / 2)^{n-1}$.

Proof: Let $f_{1}$ be the function constructed in Theorem 4.6 and set $f_{2}=$ $f_{3}=\cdots=f_{n}=\chi_{[0,1)}$. Then $f: \mathbf{R}^{n} \rightarrow \mathbf{C}$ defined by

$$
f\left(t_{1}, t_{2}, \ldots, t_{n}\right)=f_{1}\left(t_{1}\right) f_{2}\left(t_{2}\right) \ldots f_{n}\left(t_{n}\right)
$$

is easily seen to be equimeasurable with $f_{1}$. Thus $f^{*}=f_{1}^{*} \leq \chi_{[0,1 / z)}$. 
By Corollary 4.2 , for $j=2,3, \ldots, n$

$$
\left(\hat{f}_{j}\right)^{*}(y) \geq(3 \pi+y / 2)^{-1} \geq(3 \pi+1 / y)^{-1} \chi_{[0,1)}(y)
$$

SO

$$
\begin{aligned}
\left(\hat{f}_{1}\right)^{*}\left(y_{1}\right)\left(\hat{f}_{2}\right)^{*}\left(y_{2}\right) & \ldots\left(\hat{f}_{n}\right)^{*}\left(y_{n}\right) \\
& \geq(3 \pi+1 / 2)^{1-n}\left(\hat{f}_{1}\right)^{*}\left(y_{1}\right) \chi_{[0,1)}\left(y_{2}\right) \ldots \chi_{[0,1)}\left(y_{n}\right) .
\end{aligned}
$$

The $n$-dimensional Fourier transform of $f$ can be expressed in terms of the one-dimensional Fourier transforms of $f_{1}, f_{2}, \ldots, f_{n}$ as

$$
\hat{f}\left(x_{1}, x_{2}, \ldots, x_{n}\right)=\hat{f}_{1}\left(x_{1}\right) \hat{f}_{2}\left(x_{2}\right) \ldots \hat{f}_{n}\left(x_{n}\right)
$$

and this product is easily seen to be equimeasurable with

$$
\left(\hat{f}_{1}\right)^{*}\left(y_{1}\right)\left(\hat{f}_{2}\right)^{*}\left(y_{2}\right) \ldots\left(\hat{f}_{n}\right)^{*}\left(y_{n}\right) .
$$

Thus

$$
(\hat{f})^{*}(y) \geq(3 \pi+1 / 2)^{1-n}\left(\hat{f}_{1}\right)^{*}(y)
$$

and so

$$
\left(A \omega_{z}\right)^{1 / 2} \leq c_{1}\left(\left(\hat{f}_{1}\right)^{*}(y)+\varepsilon\right) \leq c_{1}\left((3 \pi+1 / 2)^{n-1}(\hat{f})^{*}(y)+\varepsilon\right) \leq c_{n}\left((\hat{f})^{*}(y)+\varepsilon\right)
$$

as required.

Testing over the functions we have constructed yields the following necessary condition for (1.2).

Corollary 4.8. Suppose $0<p<\infty, 0<q<\infty$ and $u$ and $v$ satisfy

$$
\left(\int_{0}^{\infty}(\hat{f})^{*}(y)^{q} u(y) d y\right)^{1 / q} \leq C\left(\int_{0}^{\infty}\left(\int_{0}^{1 / y} f^{*}\right)^{p} v(y) d y\right)^{1 / p}
$$

for all $f: \mathbf{R}^{n} \rightarrow \mathbf{C}$ in $L^{1} \cap L^{2}$. Then

$$
\sup _{A \in \mathcal{A}, z>0} \frac{\left\|A \omega_{z}\right\|_{q / 2, u}}{\left\|\omega_{z}\right\|_{p / 2, v}} \leq 4 c_{n}^{2} C^{2}
$$

where $c_{n}$ is the constant of Corollary 4.7.

Proof: Fix $A \in \mathcal{A}$ and $z>0$. Since $A$ has no infinite interval it is easy to see that $A \omega_{z}$ is non-increasing and does not vanish on $(0, \infty)$. Fix $Y>0$ and define $\varepsilon>0$ by $c_{n}(2 \varepsilon)=A \omega_{z}(Y)^{1 / 2}$. Apply Corollary 4.7 to produce a function $f$ such that $f^{*} \leq \chi_{(0,1 / z)}$ and $\left(A \omega_{z}\right)^{1 / 2} \leq c_{n}\left((\hat{f})^{*}+\varepsilon\right)^{2}$. Note that $f \in L^{1} \cap L^{2}$. For all $y \in(0, Y)$ we have

$$
c_{n}(2 \varepsilon)=A \omega_{z}(Y)^{1 / 2} \leq A \omega_{z}(y)^{1 / 2} \leq c_{n}\left((\hat{f})^{*}(y)+\varepsilon\right) .
$$


It follows that $\varepsilon \leq(\hat{f})^{*}(y)$ and we obtain

$$
A \omega_{z}(y)^{1 / 2} \leq c_{n}\left((\hat{f})^{*}(y)+\varepsilon\right) \leq 2 c_{n}(\hat{f})^{*}(y) .
$$

Now

$$
\begin{aligned}
\left(\int_{0}^{Y} A \omega_{z}(y)^{q / 2} u(y) d y\right)^{2 / q} & \leq\left(2 c_{n}\right)^{2}\left(\int_{0}^{Y}(\hat{f})^{*}(y)^{q} u(y) d y\right)^{2 / q} \\
& \leq 4 c_{n}^{2} C^{2}\left(\int_{0}^{\infty}\left(\int_{0}^{1 / y} f^{*}\right)^{p} v(y) d y\right)^{2 / p} \\
& \leq 4 c_{n}^{2} C^{2}\left(\int_{0}^{\infty}\left(\int_{0}^{1 / y} \chi(0,1 / z)\right)^{p} v(y) d y\right)^{2 / p} \\
& =4 c_{n}^{2} C^{2}\left(\int_{0}^{\infty} \omega_{z}(y)^{p / 2} v(y) d y\right)^{2 / p}
\end{aligned}
$$

Let $Y \rightarrow \infty$ to complete the proof.

\section{Necessary and sufficient conditions}

In the case $q=2$, the natural weight condition that arises from the analysis in this paper involves the level function $u^{o}$ of the weight $u$. Calculating as in the proof of Theorem 3.4 we see that for $u, v \in L^{+}$,

$$
\begin{aligned}
B_{0} & =\sup _{x>0}\left(\int_{0}^{\infty} \min \left(x^{-2}, t^{-2}\right) u^{o}(t) d t\right)^{1 / 2}\left(\int_{0}^{\infty} \min \left(x^{-p}, t^{-p}\right) v(t) d t\right)^{-1 / p} \\
& \approx \sup _{x>0}\left(x^{-2} \int_{0}^{x} u^{o}\right)^{1 / 2}\left(\int_{x}^{\infty}\left(\frac{1}{t} \int_{0}^{t} v\right) \frac{d t}{t^{p}}\right)^{-1 / p} .
\end{aligned}
$$

There is and equivalent expression in terms of $u$ rather than $u^{o}$. Let

$$
B_{1}=\sup _{x \leq y}\left(\frac{x}{y} \int_{0}^{y} u\right)^{1 / 2}\left(x^{p} \int_{x}^{\infty}\left(\frac{1}{t} \int_{0}^{t} v\right) \frac{d t}{t^{p}}\right)^{-1 / p} .
$$

The equivalence of $B_{0}$ and $B_{1}$ is an immediate consequence of Lemma 2.5.

The main result of this section is a weighted extension of the Jodeit and Torchinsky result with simple necessary and sufficient conditions on the weights. 
Theorem 5.1. Suppose $0<p \leq 2$ and $u, v \in L^{+}$. Then there exists a constant $C$ such that

$$
\left(\int_{0}^{\infty}(\hat{f})^{*}(x)^{2} u(x) d x\right)^{1 / 2} \leq C\left(\int_{0}^{\infty}\left(\int_{0}^{1 / t} f^{*}\right)^{p} v(t) d t\right)^{1 / p}
$$

holds for all $f \in L^{1} \cap L^{2}$ if and only if $B_{0}<\infty$ or equivalently $B_{1}<\infty$.

Proof: By Theorem 3.4, $B_{1}<\infty$ is sufficient to imply (5.1).

To prove the necessity of $B_{0}<\infty$ we suppose that (5.1) holds. By Corollary 4.8,

$$
\sup _{A \in \mathcal{A}, z>0} \frac{\left\|A \omega_{z}\right\|_{1, u}}{\left\|\omega_{z}\right\|_{p / 2, v}}<\infty .
$$

Recall that $\omega_{z}(t)=\min \left(z^{-2}, t^{-2}\right)$. We express $B_{0}$ in terms of $\omega_{z}$ and apply Lemma 2.2 to get

$$
B_{0}^{2}=\sup _{z>0} \frac{\int_{0}^{\infty} \omega_{z} u^{o}}{\left\|\omega_{z}\right\|_{p / 2, v}}=\sup _{A \in \mathcal{A}, z>0} \frac{\int_{0}^{\infty}\left(A \omega_{z}\right) u}{\left\|\omega_{z}\right\|_{p / 2, v}}<\infty .
$$

This completes the proof.

As a consequence we can characterize the boundedness of the Fourier transform as a map between weighted Lorentz spaces.

Corollary 5.2. Suppose $0<p \leq 2$ and $u, v \in L^{+}$. The following are equivalent:

$$
\begin{aligned}
& \mathcal{F}: \Gamma_{p}(w) \rightarrow \Lambda_{2}(u), \\
& \mathcal{F}: \Gamma_{p}(w) \rightarrow \Lambda_{2}\left(u^{o}\right), \\
& \mathcal{F}: \Gamma_{p}(w) \rightarrow \Gamma_{2}\left(u^{o}\right), \\
& \mathcal{F}: \Gamma_{p}(w) \rightarrow \Gamma_{2}(u),
\end{aligned}
$$

and

$$
\sup _{x \leq y}\left(\frac{x}{y} \int_{0}^{y} u\right)^{1 / 2}\left(x^{p} \int_{0}^{1 / x}\left(t^{p} \int_{t}^{\infty} w(s) \frac{d s}{s^{p}}\right) \frac{d t}{t}\right)^{-1 / p}<\infty .
$$

Proof: We show that all the statements are equivalent to the condition $B_{0}<\infty$ with $v(t)=t^{p-2} w(1 / t)$.

Theorem 5.1 shows that $B_{0}<\infty$ is equivalent to (5.1) which is readily reduced to the inequality

$$
\|\hat{f}\|_{\Lambda_{2}(u)} \leq C\|f\|_{\Gamma_{p}(w)} .
$$

Thus we wee that $B_{0}<\infty$ is equivalent to $\mathcal{F}: \Gamma_{p}(w) \rightarrow \Lambda_{2}(u)$. 
Since $u^{o}$ is non-increasing we have $\left(u^{o}\right)^{o}=u^{o}$ and it follows that $B_{0}<\infty$ for the pair $(u, v)$ if and only if it is finite for the pair $\left(u^{o}, v\right)$. We apply Theorem 5.1 with $u$ replaced by $u^{o}$ to show that $B_{0}<\infty$ is equivalent to $\mathcal{F}: \Gamma_{p}(w) \rightarrow \Lambda_{2}\left(u^{o}\right)$.

It follows easily from $\left[\mathbf{1 1}\right.$, Theorem 4] that, since $u^{o}$ is non-increasing, $\Lambda_{2}\left(u^{o}\right)=\Gamma_{2}\left(u^{o}\right)$ with equivalent norms. Therefore, $\mathcal{F}: \Gamma_{p}(w) \rightarrow \Gamma_{2}\left(u^{o}\right)$ is also equivalent to $B_{0}<\infty$.

For any $f$,

$$
\int_{0}^{\infty}\left(f^{*}\right)^{2} u \leq \int_{0}^{\infty}\left(f^{* *}\right)^{2} u \leq \int_{0}^{\infty}\left(f^{* *}\right)^{2} u^{o}
$$

so $\Gamma_{2}\left(u^{o}\right) \subseteq \Gamma_{2}(u) \subseteq \Lambda_{2}(u)$. Thus $\mathcal{F}: \Gamma_{p}(w) \rightarrow \Gamma_{2}(u)$ is equivalent to $B_{0}<\infty$ as well.

Lemma 2.5 shows that $B_{0}<\infty$ if and only $B_{1}<\infty$ and the substitution $t \rightarrow 1 / t$ in the second factor reduces $B_{1}<\infty$ to (5.2). This completes the proof.

As a map between these weighted Lorentz spaces, no operator of type $(1, \infty)$ and $(2,2)$ behaves worse than the Fourier transform. Our final result makes this statement precise.

Corollary 5.3. Suppose that $0<p \leq 2$ and $u, w \in L^{+}$. If $\mathcal{F}: \Gamma_{p}(w) \rightarrow$ $\Lambda_{2}(u)$ then $T: \Gamma_{p}(w) \rightarrow \Lambda_{2}(u)$ for any operator $T$ of type $(1, \infty)$ and $(2,2)$.

Proof: If $\mathcal{F}: \Gamma_{p}(w) \rightarrow \Lambda_{2}(u)$ then $B_{0}<\infty$. (Again we take $v(t)=$ $t^{p-2} w(1 / t)$.) In the proof of Theorem 5.1 we saw that this is equivalent to (5.2). By Corollary 3.2, we have $T: \Gamma_{p}(w) \rightarrow \Lambda_{2}(u)$ for any operator $T$ of type $(1, \infty)$ and $(2,2)$.

\section{References}

[1] J. J. Benedetto And H. P. Heinig, Weighted Hardy spaces and the Laplace transform, in: "Harmonic analysis" (Cortona, 1982), Lecture Notes in Math. 992, Springer, Berlin, 1983, pp. 240-277.

[2] J. J. Benedetto, H. P. Heinig and R. Johnson, Weighted Hardy spaces and the Laplace transform. II, Math. Nachr. 132 (1987), 29-55.

[3] C. Bennett and R. Sharpley, "Interpolation of operators", Pure and Applied Mathematics 129, Academic Press, Inc., Boston, MA, 1988.

[4] A. Gogatishvili And L. Pick, Discretization and antidiscretization of rearrangement invariant norms, Publ. Mat. (to appear). 
[5] M. L. Gol'dman, H. P. Heinig and V. D. Stepanov, On the principle of duality in Lorentz spaces, Canad. J. Math. 48(5) (1996), 959-979.

[6] I. Halperin, Function spaces, Canadian J. Math. 5 (1953), 273-288.

[7] H. P. Heinig, Weighted Fourier inequalities, new proof and generalitzation, (to appear).

[8] M. Jodeit, JR. And A. Torchinsky, Inequalities for Fourier transforms, Studia Math. 37 (1970/71), 245-276.

[9] G. G. Lorentz, "Bernstein polynomials", Mathematical Expositions 8, University of Toronto Press, Toronto, 1953.

[10] L. Maligranda, Weighted inequalities for quasi-monotone functions, J. London Math. Soc. (2) 57(2) (1998), 363-370.

[11] E. T. SAWYer, Boundedness of classical operators on classical Lorentz spaces, Studia Math. 96(2) (1990), 145-158.

[12] G. Sinnamon, Spaces defined by the level function and their duals, Studia Math. 111(1) (1994), 19-52.

[13] G. Sinnamon, Interpolation of spaces defined by the level function, in: "Harmonic analysis" (Sendai, 1990), ICM-90 Satell. Conf. Proc., Springer, Tokyo, 1991, pp. 190-193.

[14] G. Sinnamon, The level function in rearrangement invariant spaces, Publ. Mat. 45(1) (2001), 175-198.

[15] G. Sinnamon, Embeddings of concave functions and duals of Lorentz spaces, Publ. Mat. 46(2) (2002), 489-515.

[16] V. D. Stepanov, Integral operators on the cone of monotone functions, J. London Math. Soc. (2) 48(3) (1993), 465-487.

Department of Mathematics

University of Western Ontario

London, Ontario, N6A 5B7

Canada

E-mail address: sinnamon@uwo.ca

Rebut el 13 de juny de 2001. 